\title{
Acoustic Impedance of an Annular Piston Wobbling in a Flat Screen Around a Semi-infinite Circular Cylinder
}

\author{
D. PIECZONKA ${ }^{a}$, W.P. RdZANEK ${ }^{b, *}$ AND W.J. RDZANEK ${ }^{b}$ \\ ${ }^{a}$ Stylem, 36-001 Trzebownisko 614, Poland \\ ${ }^{b}$ Department of Acoustics, Institute of Physics, University of Rzeszów \\ Rejtana 16A, 35-310 Rzeszów, Poland
}

\begin{abstract}
A rigorous solution is presented for the problem of sound radiation by an oscillating and wobbling annular piston embedded concentrically in a perpendicular flat screen surrounding a semi-infinite circular cylindrical baffle. Two forms of the Green's function of the considered region are used. The acoustic impedance is presented in its integral form useful for numerical calculations which enable studying the effect of the acoustic waves scattering on the cylindrical baffle and the asymmetry of vibration velocity on the piston on the resultant acoustic impedance of the wobbling piston. It is shown that in the case of the vibrating piston under consideration, the reciprocity of acoustic impedance related to two modes of rigid body motion, oscillating and wobbling, does not occur.
\end{abstract}

DOI: 10.12693 /APhysPolA.123.1078

PACS: 43.20.Bi, 43.20.El, 43.20.Fn, 43.20.Rz

\section{Introduction}

From the practical point of view, phenomena related to sound radiation generated by vibrating pistons, membranes, and plates, both circular and elliptical, embedded in a flat acoustically rigid screen is still interesting. A number of studies deals with such problems. Wyrzykowska investigated sound radiation by oscillating pistons, circular, annular and rectangular, in the range of low frequencies [1]. Pritchard analyzed the acoustic mutual impedance between oscillating pistons arranged as a hexagonal system of sources [2]. Porter investigated the self- and mutual impedance of circular radiators of the uniform vibration velocity distribution [3]. Thompson Jr. calculated the self and mutual impedance of oscillating annular and elliptical pistons [4]. Stepanishen presented the impulse response and the acoustic impedance of the annular piston [5]. Mellow and Kärkkäinen calculated the acoustic impedance of a vibrating disk in a circular baffle of finite dimensions [6]. Mellow presented the acoustic impedance of a vibrating resilient disk in the range of low frequencies [7]. A number of further studies concern the use of a variety of theoretical and experimental methods for determining the acoustic impedance of circular and rectangular plates and membranes [8-15].

For practical reasons, the solutions to asymmetric problems are particularly important. Mangulis [16] was the first who published results of the acoustic impedance of oscillating and wobbling circular piston embedded in a flat rigid screen.

Rdzanek et al. presented the Green's function of the region located above a flat screen around a circular cylindrical baffle [17]. On the basis of results presented in this

*corresponding author; e-mail: wprdzank@univ.rzeszow.pl study, Rdzanek, Rdzanek, and Pieczonka proposed the integral formulations of acoustic impedance of an oscillating annular piston embedded in a flat screen around a semi infinite circular cylindrical baffle [8].

The study of Mangulis [16] was the only one of the studies mentioned above that concerned the sound radiation of a circular piston wobbling in a flat screen. To the best of the authors' knowledge, the acoustic impedance of a wobbling annular piston embedded in a flat screen concentrically around a circular cylindrical baffle has not been presented so far. The rigorous solution to this problem is proposed in this study.

\section{Theoretical analysis}

An asymmetric steady-state vibration velocity distribution was assumed on the surface of an annular piston. The velocity component $\left.\left.v\right|_{z_{0}=0} \equiv \boldsymbol{n} \cdot \boldsymbol{v}\right|_{z_{0}=0}$ normal to the surface of the flat screen was adopted as follows:

$$
v\left(r_{0}, \varphi_{0}, t\right)=\left[v_{0}+v_{1} \frac{r_{0}}{a_{1}} \cos \left(\beta-\varphi_{0}\right)\right] e^{-\mathrm{i} \omega t},
$$

where $r_{0}, \varphi_{0}$ are the polar coordinates of the piston's point, $\beta=$ const is the rotation angle of the normal to the nodal diameter of the piston's wobbling, $v_{0}=\left|v_{0}\right|$ is the velocity amplitude of the piston's transverse oscillations (the zero initial phase assumed), $v_{1}=\left|v_{1}\right| e^{\mathrm{i} \alpha}$ is the velocity amplitude of wobbling of the point located at the piston's inner edge of coordinates $r_{0}=a_{1}$ and $\varphi_{0}=\beta$, $\alpha$ is the initial phase difference between the transverse oscillation velocity and the wobbling velocity, $\omega=k c$ is the vibrations' pulsation, $k$ is the wavenumber, $c$ is the speed of sound in air, $\mathrm{i}^{2}=-1$.

Equation (1) indicates that the piston vibrations are composed of transverse oscillations (Fig. 1a) and wobbling (Fig. 1b) with the initial phase difference equal to $\alpha$. The velocity amplitude of wobbling at the point at the outer piston's edge, with coordinates $r_{0}=a_{2}$ and 
$\varphi_{0}=\beta$, equals obviously to $v_{1} a_{2} / a_{1}$. The nodal line of the piston's wobbling presented is in the top view in Fig. 2. Mangulis, in his study [16], assumed a similar vibration velocity for a circular piston with a difference consisting in that he assumed $\beta=0$ and $v_{w}$ being the velocity amplitude of wobbling of the point located at the outer edge of a circular piston.

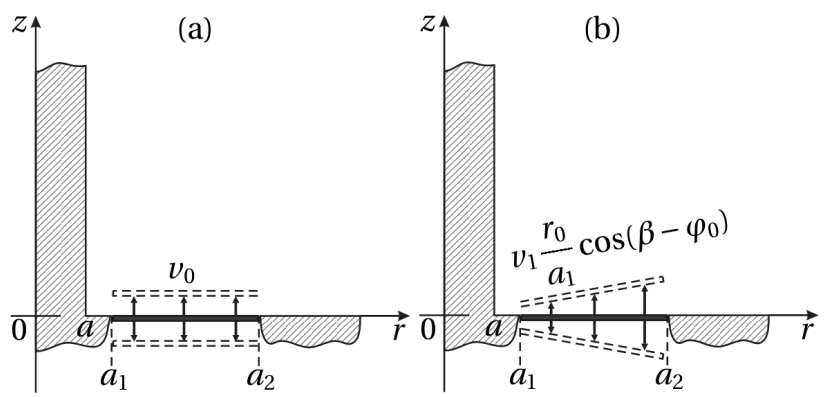

Fig. 1. The rigid body modes of an annular piston located on a flat screen around a circular cylindrical baffle of radius $a$ : (a) oscillating, (b) wobbling. Solid line - piston's equilibrium position; dashed line - piston's maximum deflection.

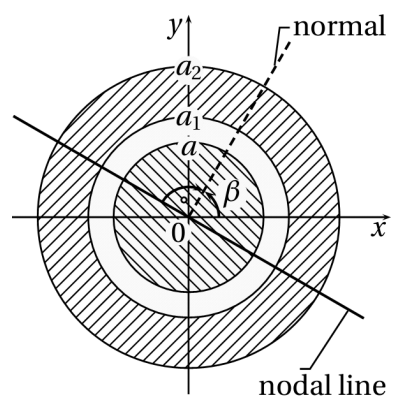

Fig. 2. The configuration of an annular piston (the radius of inner edge $a_{1}$ and the radius of outer edge $a_{2}$ ) located on a flat screen around a semi infinite circular cylindrical baffle (the radius $a$ ). Solid line - the nodal line of the wobbling piston; dashed line - the normal to the nodal line.

The Green function presented in [17] was used to solve the wave equation and calculate the acoustic pressure amplitude. It was assumed that the resultant vibration velocity of the piston is small $\left(\left|v_{0}\right|,\left|v_{1}\right| \ll c\right)$. The Green's function $G\left(\boldsymbol{r} \mid \boldsymbol{r}_{0}\right)$ satisfies the Neumann's boundary conditions at both baffles (the flat screen and the semi infinite circular cylindrical baffle),

$$
\begin{gathered}
\left.\frac{\partial G\left(r, \varphi, z \mid r_{0}, \varphi_{0}, z_{0}\right)}{\partial r}\right|_{r=a}=0, \\
\left.\frac{\partial G\left(r, \varphi, z \mid r_{0}, \varphi_{0}, z_{0}\right)}{\partial z}\right|_{z=0}=0,
\end{gathered}
$$

where $\left(r_{0}, \varphi_{0}, z_{0}\right)$ are the source's point cylindrical coordinates, and $(r, \varphi, z)$ are the observation point cylindrical coordinates. The tightened Sommerfeld's radiation con- ditions (cf. $[18,19])$ are also satisfied at an infinitely large distance from the source $\left(0<a<a_{1}<a_{2} \ll \sqrt{r^{2}+z^{2}}\right)$. The Green's function assumes the form of

$$
\begin{aligned}
G & \left(r, \varphi, z \mid r_{0}, \varphi_{0}, z_{0}\right) \\
& =\sum_{m=0}^{\infty} \epsilon_{m} \cos m\left(\varphi-\varphi_{0}\right) G_{m}\left(r, z \mid r_{0}, z_{0}\right),
\end{aligned}
$$

where

$$
\begin{aligned}
& \epsilon_{m}=\left\{\begin{array}{ll}
1 ; & m=0, \\
2 ; & m=1,2,3, \ldots,
\end{array},\right. \\
& G_{m}\left(r, z \mid r_{0}, z_{0}\right)=\frac{\mathrm{i} k}{2 \pi} \int_{0}^{\infty} \mathcal{F}_{m}\left(r \mid r_{0}\right) \cos (k z u) \\
& \times \cos \left(k z_{0} u\right) \mathrm{d} u, \\
& \mathcal{F}_{m}\left(r \mid r_{0}\right)=\frac{H_{m}^{(1)}\left(k r_{0} \sqrt{1-u^{2}}\right)}{H_{m}^{(1) \prime}\left(k a \sqrt{1-u^{2}}\right)} \\
& \times\left[J_{m}\left(k r \sqrt{1-u^{2}}\right) H_{m}^{(1) \prime}\left(k a \sqrt{1-u^{2}}\right)\right. \\
& \left.-H_{m}^{(1)}\left(k r \sqrt{1-u^{2}}\right) J_{m}^{\prime}\left(k a \sqrt{1-u^{2}}\right)\right], \\
& a \leqslant r \leqslant r_{0}<\infty \text {, } \\
& \mathcal{F}_{m}\left(r \mid r_{0}\right)=\frac{H_{m}^{(1)}\left(k r \sqrt{1-u^{2}}\right)}{H_{m}^{(1) \prime}\left(k a \sqrt{1-u^{2}}\right)} \\
& \times\left[J_{m}\left(k r_{0} \sqrt{1-u^{2}}\right) H_{m}^{(1) \prime}\left(k a \sqrt{1-u^{2}}\right)\right. \\
& \left.\times-H_{m}^{(1)}\left(k r_{0} \sqrt{1-u^{2}}\right) J_{m}^{\prime}\left(k a \sqrt{1-u^{2}}\right)\right] \text {, } \\
& a \leqslant r_{0} \leqslant r<\infty .
\end{aligned}
$$

$k$ is the wavenumber, $f$ is the Cauchy's principal value, the prime sign denotes the differentiation according to the entire argument, and the integration path in Eq. (3b) is presented in Fig. 3. The two denotations of functions $\mathcal{F}$ in Eqs. (3c) and (3d) indicate that the Green's function assumes the two different forms depending on whether $r$ is larger or smaller than $r_{0}$. Both forms are necessary since they will be used later during the integration.

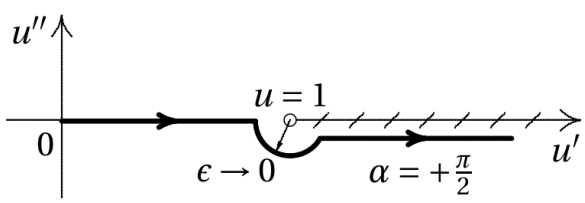

Fig. 3. The integration path in the plane of complex variable $u=u^{\prime}+\mathrm{i} u^{\prime \prime}$.

The vibration velocity in Eq. (1) and the Green's function in Eq. (2) together with the denotations in Eq. (3) were inserted to the acoustic potential equation 


$$
\phi(\boldsymbol{r})=\left.\int_{S_{0}} v\left(r_{0}, \varphi_{0}, z_{0}\right) G\left(r, \varphi, z \mid r_{0}, \varphi_{0}, z_{0}\right)\right|_{z_{0}=0} d S_{0},
$$

where $S_{0}=\pi a_{1}^{2}\left(s^{2}-1\right)$ is the area of the vibrating annular piston for $z_{0}=0$ and $s=a_{2} / a_{1}$ is the geometric parameter of the piston. Further, the integration over the interval $(0,2 \pi)$ of the angular variable $\varphi_{0}$ yields

$$
\begin{aligned}
& \phi(\boldsymbol{r})=2 \pi v_{0} a_{1} \sum_{m} \epsilon_{m} \delta_{0 m} \cos m \varphi \\
& \times \frac{1}{a_{1}} \int_{a_{1}}^{a_{2}} G_{m}\left(r, z \mid r_{0}, 0\right) r_{0} \mathrm{~d} r_{0}+\pi v_{1} a_{1} \\
& \times \sum_{m} \epsilon_{m} \delta_{1 m} \cos (\beta-m \varphi) \\
& \times \frac{1}{a_{1}^{2}} \int_{a_{1}}^{a_{2}} G_{m}\left(r, z \mid r_{0}, 0\right) r_{0}^{2} \mathrm{~d} r_{0} .
\end{aligned}
$$

The orthogonality of trigonometric functions was used during integration leading to the summation with the Kronecker delta which enables formulating the acoustic potential as follows

$$
\phi(\boldsymbol{r})=v_{0} a_{1} I_{0}(r, z)+v_{1} a_{1} I_{1}(r, z) \cos (\beta-\varphi),
$$

where

$$
\begin{aligned}
& I_{0}(r, z)=\frac{2 \pi}{a_{1}} \int_{a_{1}}^{a_{2}} G_{0}\left(r, z \mid r_{0}, 0\right) r_{0} \mathrm{~d} r_{0} \\
& =-\frac{\mathrm{i} k}{a_{1}} \int_{0}^{\infty} \frac{H_{0}^{(1)}\left(k r \sqrt{1-u^{2}}\right)}{H_{0}^{(1) \prime}\left(k a \sqrt{1-u^{2}}\right)}\left[J_{0}^{\prime}\left(k a \sqrt{1-u^{2}}\right)\right. \\
& \quad \times \int_{a_{1}}^{r} H_{0}^{(1)}\left(k r_{0} \sqrt{1-u^{2}}\right) r_{0} \mathrm{~d} r_{0}-H_{0}^{(1) \prime}\left(k a \sqrt{1-u^{2}}\right) \\
& \left.\quad \times \int_{a_{1}}^{r} J_{0}\left(k r_{0} \sqrt{1-u^{2}}\right) r_{0} \mathrm{~d} r_{0}\right] \cos (k z u) \mathrm{d} u \\
& -\frac{\mathrm{i} k}{a_{1}} \int_{0}^{\infty} \frac{1}{H_{0}^{(1) \prime}\left(k a \sqrt{1-u^{2}}\right)} \int_{r}^{a_{2}} H_{0}^{(1)}\left(k r_{0} \sqrt{1-u^{2}}\right) r_{0} \mathrm{~d} r_{0} \\
& \quad \times\left[J_{0}^{\prime}\left(k a \sqrt{1-u^{2}}\right) H_{0}^{(1)}\left(k r \sqrt{1-u^{2}}\right)\right. \\
& \left.\quad-H_{0}^{(1) \prime}\left(k a \sqrt{1-u^{2}}\right) J_{0}\left(k r \sqrt{1-u^{2}}\right)\right] \cos (k z u) \mathrm{d} u \\
& I_{1}(r, z)=\frac{2 \pi}{a_{1}^{2}} \int_{a_{1}}^{a_{2}} G_{1}\left(r, z \mid r_{0}, 0\right) r_{0}^{2} \mathrm{~d} r_{0} \\
& \quad=-\frac{\mathrm{i} k}{a_{1}^{2}} \int_{0}^{\infty} \frac{H_{1}^{(1)}\left(k r \sqrt{1-u^{2}}\right)}{H_{1}^{(1) \prime}\left(k a \sqrt{1-u^{2}}\right)}\left[J_{1}^{\prime}\left(k a \sqrt{1-u^{2}}\right)\right. \\
& \quad \times \int_{a_{1}}^{r} H_{1}^{(1)}\left(k r_{0} \sqrt{1-u^{2}}\right) r_{0}^{2} \mathrm{~d} r_{0}-H_{1}^{(1) \prime}\left(k a \sqrt{1-u^{2}}\right)
\end{aligned}
$$

$$
\begin{aligned}
&\left.\times \int_{a_{1}}^{r} J_{1}\left(k r_{0} \sqrt{1-u^{2}}\right) r_{0}^{2} \mathrm{~d} r_{0}\right] \cos (k z u) \mathrm{d} u \\
&-\frac{\mathrm{i} k}{a_{1}^{2}} \int_{0}^{\infty} \frac{1}{H_{1}^{(1) \prime}\left(k a \sqrt{1-u^{2}}\right)} \int_{r}^{a_{2}} H_{1}^{(1)}\left(k r_{0} \sqrt{1-u^{2}}\right) r_{0}^{2} \mathrm{~d} r_{0} \\
& \quad \times\left[J_{1}^{\prime}\left(k a \sqrt{1-u^{2}}\right) H_{1}^{(1)}\left(k r \sqrt{1-u^{2}}\right)\right. \\
&\left.-H_{1}^{(1) \prime}\left(k a \sqrt{1-u^{2}}\right) J_{1}\left(k r \sqrt{1-u^{2}}\right)\right] \cos (k z u) \mathrm{d} u .
\end{aligned}
$$

It is worth noticing that the integration over the interval $\left(a_{1}, a_{2}\right)$ of the radial variable $r_{0}$ of the source's point in Eqs. (7) was divided to two integrals calculated separately, one over the interval $\left(a_{1}, r\right)$ (where Eq. (3d) was used) and the another over the interval $\left(r, a_{2}\right)$ (where Eq. (3c) was used). Therefore, both forms of the Green's function (presented in [17]) were used for the considered problem. This approach enables solving the problem and finding the acoustic potential. For this purpose, the following Wronskian was used [20,21]

$$
H_{1}^{(1)}(z) J_{0}(z)-H_{0}^{(1)}(z) J_{1}(z)=-\frac{2 \mathrm{i}}{\pi z},
$$

the following integrals

$$
\begin{aligned}
& \int^{r} \mathcal{C}_{0}(k r) r \mathrm{~d} r=\frac{r}{k} \mathcal{C}_{1}(k r), \\
& \int^{r} \mathcal{C}_{1}(k r) r^{2} \mathrm{~d} r=\frac{r^{2}}{k} \mathcal{C}_{2}(k r),
\end{aligned}
$$

where $\mathcal{C}_{\nu}$ is a cylindrical function for $\nu=0,1,2$, and the Hilbert transform of the cosine function [22]

$$
\frac{2}{\pi} \int_{0}^{\infty} \frac{\cos (k z u) \mathrm{d} u}{1-u^{2}}=\sin (k z)
$$

valid for $0<k$. This enables formulating the integrals in Eqs. (7) in the form of the following single integrals:

$$
\begin{aligned}
& I_{0}(r, z)=-\frac{\sin (k z)}{k a_{1}} \\
& +\mathrm{i} \int_{0}^{\infty}\left\{s H_{1}^{(1)}\left(k a_{2} \sqrt{1-u^{2}}\right) J_{0}\left(k r \sqrt{1-u^{2}}\right)\right. \\
& -J_{1}\left(k a_{1} \sqrt{1-u^{2}}\right) H_{0}^{(1)}\left(k r \sqrt{1-u^{2}}\right) \\
& -\frac{J_{0}^{\prime}\left(k a \sqrt{1-u^{2}}\right)}{H_{0}^{(1) \prime}\left(k a \sqrt{1-u^{2}}\right)}\left[s H_{1}^{(1)}\left(k a_{2} \sqrt{1-u^{2}}\right)\right. \\
& \left.\left.-H_{1}^{(1)}\left(k a_{1} \sqrt{1-u^{2}}\right)\right] H_{0}^{(1)}\left(k r \sqrt{1-u^{2}}\right)\right\} \\
& \times \frac{\cos (k z u) \mathrm{d} u}{\sqrt{1-u^{2}}}
\end{aligned}
$$




$$
\begin{aligned}
& I_{1}(r, z)=-\frac{r}{a_{1}} \frac{\sin k z}{k a_{1}} \\
& +\mathrm{i} \int_{0}^{\infty}\left\{s^{2} H_{2}^{(1)}\left(k a_{2} \sqrt{1-u^{2}}\right) J_{1}\left(k r \sqrt{1-u^{2}}\right)\right. \\
& -J_{2}\left(k a_{1} \sqrt{1-u^{2}}\right) H_{1}^{(1)}\left(k r \sqrt{1-u^{2}}\right) \\
& -\frac{J_{1}^{\prime}\left(k a \sqrt{1-u^{2}}\right)}{H_{1}^{(1) \prime}\left(k a \sqrt{1-u^{2}}\right)}\left[s^{2} H_{2}^{(1)}\left(k a_{2} \sqrt{1-u^{2}}\right)\right. \\
& \left.\left.-H_{2}^{(1)}\left(k a_{1} \sqrt{1-u^{2}}\right)\right] H_{1}^{(1)}\left(k r \sqrt{1-u^{2}}\right)\right\} \\
& \quad \times \frac{\cos (k z u) \mathrm{d} u}{\sqrt{1-u^{2}}} .
\end{aligned}
$$

It is worth noticing that in the above integrals

$$
\lim _{z \rightarrow 0} \frac{J_{0}^{\prime}(z)}{H_{0}^{(1) \prime}(z)}=0, \quad \lim _{z \rightarrow 0} \frac{J_{1}^{\prime}(z)}{H_{1}^{(1) \prime}(z)}=0,
$$

where it was assumed that $z=k a \sqrt{1-u^{2}}$. This means that the terms containing such quotients represent the correction for the semi-infinite circular cylindrical baffle for $0<a<a_{1}$, whereas when there is no such baffle, their limits are equal to zero when $a \rightarrow 0$.

The knowledge of the acoustic potential enables expressing the acoustic pressure amplitude, for the time harmonic steady state vibrations, in the form

$$
p=\varrho_{0} \frac{\partial \phi}{\partial t}=-\mathrm{i} k \varrho_{0} c \phi
$$

in the cylindrical coordinates, where $p \equiv p(r, \varphi, z, t)=$ $p(r, \varphi, z) e^{-i \omega t}$ and $\phi \equiv \phi(r, \varphi, z, t)=\phi(r, \varphi, z) e^{-i \omega t}$. The acoustic potential in Eq. (6) was inserted to Eq. (12), and the integrals in Eqs. (10) used giving the acoustic pressure amplitude useful for some further calculations of the acoustic power in the impedance approach. The resultant acoustic pressure above the piston $\left(a_{1} \leqslant r \leqslant a_{2}\right.$, $0 \leqslant z<\infty)$

$$
\begin{aligned}
& p(r, \varphi, z)=-\mathrm{i} k a_{1} \varrho_{0} c\left[v_{0} I_{0}(r, z)\right. \\
& \left.\quad+v_{1} I_{1}(r, z) \cos (\beta-\varphi)\right]
\end{aligned}
$$

is the superposition of the two components. The first of them does not depend on the angular variable $\varphi$ and is associated only with the axisymmetric transverse oscillations of the piston (it contains the integral $I_{0}$ and cylindrical functions of the orders zero and one). The second depends on the angular variable $\varphi$ and is associated with the asymmetric wobbling of the piston (it contains the integral $I_{1}$ and the cylindrical functions of the one order higher than those of the axisymmetric component).

The time-averaged acoustic power

$$
\begin{aligned}
\Pi & =\left.\frac{1}{2} \int_{S} p\right|_{z=0} v^{*} \mathrm{~d} S \\
& =\frac{1}{2} \int_{a_{1}}^{a_{2}} \int_{0}^{2 \pi} p(r, \varphi, 0) v^{*}(r, \varphi) \mathrm{d} \varphi r \mathrm{~d} r
\end{aligned}
$$

was calculated in the impedance approach where $S \equiv S_{0}$ is the area of the piston. After integrating over the angular variable $\varphi$ it was obtained that

$$
\Pi=\varrho_{0} c \pi a_{1}^{2}\left(\bar{I}_{0}\left|v_{0}\right|^{2}+\frac{1}{2} \bar{I}_{1}\left|v_{1}\right|^{2}\right),
$$

where

$$
\bar{I}_{n}=-\frac{\mathrm{i} k}{a_{1}^{n+1}} \int_{a_{1}}^{a_{2}} I_{n}(r, 0) r^{n+1} \mathrm{~d} r
$$

for $n=0,1$. The expression in Eq. (15) together with the integrals in Eqs. (16) represents the time averaged acoustic power in its spectral form. It is worth noticing, that this expression is the superposition of the acoustic power of the transversely oscillating piston (the term containing the integral $\bar{I}_{0}$ ) and of the acoustic power of the wobbling piston (the term containing the integral $\bar{I}_{1}$ ), similarly as in the case of the acoustic pressure (cf. Eq. (13)). The following facts are essential that there is no mutual power between the two rigid body modes due to $\int_{0}^{2 \pi} \cos (\beta-\varphi) \mathrm{d} \varphi=0$ for $\beta=$ const, and that the acoustic power does not depend on the value of the constant $\beta$. For the same reason, the acoustic power does not depend on the difference $\alpha$ of the initial phases of oscillating and wobbling of the piston.

Further, the integration over the radial variable $r$ was performed giving

$$
\bar{I}_{n}=\int_{0}^{\infty} \frac{F_{n}\left(s, s_{1}, k a_{1} \sqrt{1-u^{2}}\right) \mathrm{d} u}{1-u^{2}}
$$

for $n=0,1$, where

$$
\begin{aligned}
& F_{n}\left(s, s_{1}, z\right)=s^{n+1} H_{n+1}^{(1)}(s z) \hat{J}_{n+1}(s, z)-\hat{H}_{n+1}^{(1)}(s, z) \\
& \quad \times\left[J_{n+1}(z)+\frac{J_{n}^{\prime}\left(s_{1} z\right)}{H_{n}^{(1) \prime}\left(s_{1} z\right)} \hat{H}_{n+1}^{(1)}(s, z)\right],
\end{aligned}
$$

$s=a_{2} / a_{1}$ is the geometric parameter of the annular piston, $s_{1}=a / a_{1}$ is the normalized radius of the circular cylindrical baffle, and the following denotation was introduced:

$$
\hat{\mathcal{C}}_{n}(s, z)=s^{n} \mathcal{C}(s z)-\mathcal{C}_{n}(z) .
$$

The quotient of cylindrical functions in Eq. (17b), such as in Eq. (11), represents the influence of the circular cylindrical baffle on the acoustic power similarly as in the case of the acoustic pressure amplitude (cf. Eqs. (10)). Further, the following reference acoustic power was formulated:

$$
\Pi^{(\infty)}=\frac{1}{2} \varrho_{0} c S\left\langle|v|^{2}\right\rangle,
$$

where

$$
\left\langle|v|^{2}\right\rangle=\frac{1}{S} \int_{S}|v(r, \varphi)|^{2} \mathrm{~d} S=\left|v_{0}\right|^{2}\left[1+\frac{1}{4} \kappa^{2}\left(s^{2}+1\right)\right],
$$

$S=\pi a_{1}^{2}\left(s^{2}-1\right)$ is the piston's area, and $\kappa=\left|v_{1}\right| /\left|v_{0}\right|$ is the quotient of the velocity moduli of oscillations and wobbling. The normalized acoustic impedance was formulated as follows 


$$
\zeta=\frac{\Pi}{\Pi^{(\infty)}}=\theta-\mathrm{i} \chi=\frac{\zeta_{0}+\frac{\kappa^{2}}{4}\left(s^{2}+1\right) \zeta_{1}}{1+\frac{\kappa^{2}}{4}\left(s^{2}+1\right)},
$$

where

$$
\zeta_{0}=\frac{2 \bar{I}_{0}}{s^{2}-1}, \quad \zeta_{1}=\frac{4 \bar{I}_{1}}{s^{4}-1},
$$

$\theta=\Re \zeta$ is the normalized acoustic resistance (represents the acoustic energy that is radiated by the source to the far field and does not return), $\chi=-\Im \zeta$ is the normalized acoustic reactance (represents the acoustic energy fluctuating in the near field), $\zeta_{0}$ is the normalized acoustic impedance of the piston that only oscillates axisymmetrically, whereas $\zeta_{1}$ is the acoustic impedance of the same piston that is only wobbling, and the integrals $\bar{I}_{0}$ and $\bar{I}_{1}$ were presented in Eq. (17a).

\section{Numerical analysis}

For the purpose of numerical analysis, it is convenient to perform the following substitution $x=\sqrt{1-u^{2}}$ and to use the Cauchy's theorem, to present the values from Eqs. (21b) in the form of

$$
\begin{aligned}
& \zeta_{0}=1-\frac{2 \mathrm{i}}{s^{2}-1} \int_{1}^{\infty} \frac{F_{0}\left(s, s_{1}, k a_{1} x\right) \mathrm{d} x}{x \sqrt{x^{2}-1}}, \\
& \zeta_{1}=1-\frac{4 \mathrm{i}}{s^{4}-1} \int_{1}^{\infty} \frac{F_{1}\left(s, s_{1}, k a_{1} x\right) \mathrm{d} x}{x \sqrt{x^{2}-1}} .
\end{aligned}
$$

The above integrals have singularities only at the limits of integration interval and the integrals are useful for numerical integration. The method of achieving such similar integrals was presented in detail in $[8,9,10,11]$ and therefore it was not discussed in this study. Additionally, the exact formulations of the normalized acoustic impedance of the oscillating and wobbling circular piston embedded in the flat rigid baffle can be used. The formulation suitable for the oscillating circular piston has been originally presented by Rayleigh [23]

$$
\begin{aligned}
& Z_{0}(z)=1-2 \mathrm{i} \int_{1}^{\infty} \frac{J_{1}(z x) H_{1}^{(1)}(z x) \mathrm{d} x}{x \sqrt{x^{2}-1}} \\
& =1-\frac{1}{z} J_{1}(2 z)-\frac{\mathrm{i}}{z} \boldsymbol{H}_{1}(2 z),
\end{aligned}
$$

whereas the formulation suitable for the wobbling circular piston has been reported by Mangulis [16]

$$
\begin{aligned}
& Z_{1}(z)=1-4 \mathrm{i} \int_{1}^{\infty} \frac{J_{2}(z x) H_{2}^{(1)}(z x) \mathrm{d} x}{x \sqrt{x^{2}-1}} \\
& =1+\frac{2}{z}\left[J_{1}(2 z)-\frac{2}{z} J_{2}(2 z)\right] \\
& +\frac{2 \mathrm{i}}{z}\left[\boldsymbol{H}_{1}(2 z)-\frac{3}{z} \boldsymbol{H}_{2}(2 z)\right],
\end{aligned}
$$

where $\boldsymbol{H}_{n}$ is the Struve function of the order 1,2 [24], and $z$ is to be replaced by either $k a_{1}$ or $s k a_{1}$. The application of above formulations enables expressing Eqs. (21) in the form

$$
\zeta_{0}=1-\frac{s^{2}\left[1-Z_{0}\left(s k a_{1}\right)\right]+1-Z_{0}(k a 1)}{s^{2}-1}
$$

$$
\begin{gathered}
-\frac{2 \mathrm{i}}{s^{2}-1} \int_{1}^{\infty} \frac{\tilde{F}_{0}\left(s, s_{1}, k a_{1} x\right) \mathrm{d} x}{x \sqrt{x^{2}-1}}, \\
\zeta_{1}=1-\frac{s^{4}\left[1-Z_{1}\left(s k a_{1}\right)\right]+1-Z_{1}(k a 1)}{s^{4}-1} \\
-\frac{4 \mathrm{i}}{s^{4}-1} \int_{1}^{\infty} \frac{\tilde{F}_{1}\left(s, s_{1}, k a_{1} x\right) \mathrm{d} x}{x \sqrt{x^{2}-1}},
\end{gathered}
$$

where

$$
\begin{aligned}
& \tilde{F}_{n}\left(s, s_{1}, z\right)=-2 s^{n+1} H_{n+1}^{(1)}(s z) J_{n+1}(z) \\
& \times-\frac{J_{n}^{\prime}\left(s_{1} z\right)}{H_{n}^{(1) \prime}\left(s_{1} z\right)}\left[\hat{H}_{n+1}^{(1)}(s, z)\right]^{2}
\end{aligned}
$$

is the reduced version of Eq. (17b).

Applying Eqs. (20) and (22)-(24) enabled performing a number of numerical analyses and examining the impact of the circular cylindrical baffle on sound radiation as well. It was assumed for this purpose that the mean square of oscillation velocity of the piston is equal to the mean square of its wobbling velocity,

$$
\int_{S}\left|v_{0}\right|^{2} d S=\int_{S}\left|v_{1}(r, \varphi)\right|^{2} \mathrm{~d} S
$$

where $v_{1}(r, \varphi)=\left(r / a_{1}\right)\left|v_{1}\right||\cos (\beta-\varphi)|$, which led to the following condition

$$
\kappa=\frac{2}{\sqrt{s^{2}+1}}
$$

and enabled comparing the acoustic impedance of the piston oscillating only - and the acoustic impedance of the piston wobbling only. This assumption led to the following formulation of the normalized superimposed acoustic impedance:

$$
\zeta=\frac{1}{2}\left(\zeta_{0}+\zeta_{1}\right)
$$

being the complex arithmetic mean value of both - the acoustic impedance $\zeta_{0}$ the piston oscillating only - and the acoustic impedance $\zeta_{1}$ the piston wobbling only.

The curves of the normalized acoustic impedance of oscillating and wobbling piston are presented in Fig. 4 for different values of the quotient $s_{1}=a_{2} / a_{1}$. In the case of no circular cylindrical baffle $\left(s_{1}=0.0\right)$, this quantity is a complex superposition of the acoustic impedance of both kinds of the piston's vibrations (cf. Eq. (15)). As the result, the global maximum of the acoustic resistance is shifted towards the greater values of wavenumber $k$, compared to the remaining curves in this figure. Introducing the baffle for $s_{1}=0.5$ causes the appearance of clear local maxima as the result of scattering of acoustic waves on the circular cylindrical baffle. The increase of the quotient $s_{1}$ to its maximum value equal to 1.0, causes shifting the global maximum of the acoustic resistance towards the lower values of $k$, compared to the remaining curves. This fact is important since the normalized acoustic resistance is equivalent to the sound radiation efficiency of the source. For this reason, the value of $s_{1}=1.0$ was assumed in the following figures. 


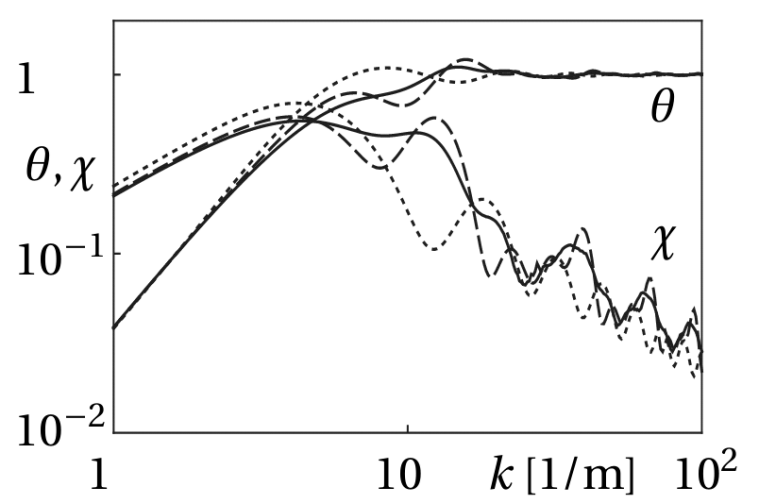

Fig. 4. The normalized acoustic impedance $\zeta=\theta-\mathrm{i} \chi$ of oscillating and wobbling piston for $s=2.0$ : (a) resistance $\theta$, (b) reactance $\chi$. Solid line $-s_{1}=0.0$; dashed line $-s_{1}=0.5$; dotted line $-s_{1}=1.0$.

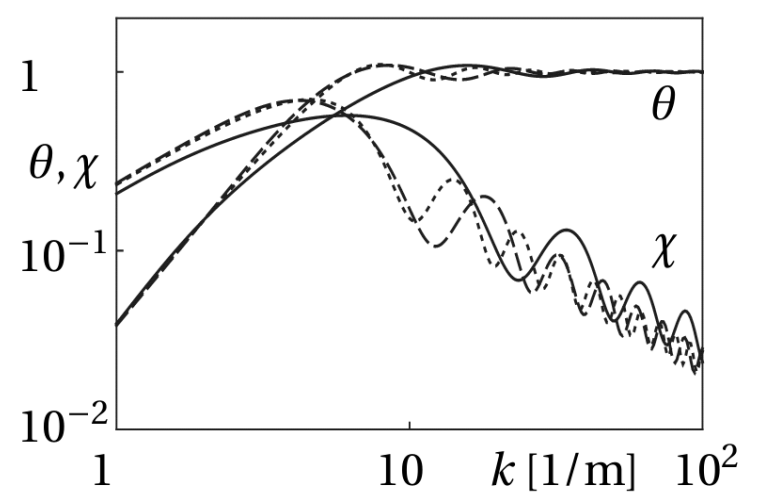

Fig. 5. The normalized acoustic impedance $\zeta=\theta-\mathrm{i} \chi$ of oscillating and wobbling piston for $s_{1}=1.0$ : (a) resistance $\theta$, (b) reactance $\chi$. Solid line $-s=1.2$; dashed line $-s=2.0 ;$ dotted line $-s=5.0$.

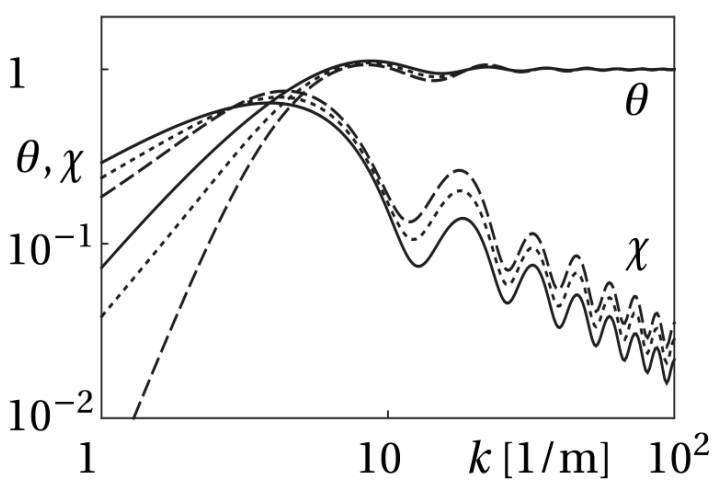

Fig. 6. The normalized acoustic impedance $\zeta=\theta-\mathrm{i} \chi$ of a vibrating piston for $s=2.0$ and $s_{1}=1.0$ : (a) resistance $\theta$, (b) reactance $\chi$. Solid line - oscillating only, $\zeta=\zeta_{0}$; dashed line - wobbling only, $\zeta=\zeta_{1}$; dotted line - oscillating and wobbling, $\zeta=\frac{1}{2}\left(\zeta_{0}+\zeta_{1}\right)$.
The normalized acoustic impedance of oscillating and wobbling piston is presented in Fig. 5 for three different values of the geometric parameter $s=a_{2} / a_{1}$. For the smallest analyzed value of $s=1.2$, the global maximum of acoustic resistance is the most strongly shifted towards the higher values of $k$ which means that the sound radiation efficiency assumes the smallest values for the smaller values of $k$. Increasing the value of $s$ up to 2.0 and more causes an increase in the sound radiation efficiency for smaller values of $k$. Redundant increasing of $s$ (up to 5.0 and more) is pointless since the cross section area of the circular cylindrical baffle becomes small compared to the area of a vibrating piston and the scattering of acoustic waves on the cylinder then becomes small for small values of $k$. Therefore, the value of $s=2.0$ was assumed in further analysis.

The acoustic impedance of a vibrating piston is presented in Fig. 6 for the piston oscillating only, for the piston wobbling only and for the piston which oscillates and wobbles at the same time. The curves presented confirm the obvious fact that the wobbling piston is a much less efficient radiator than the oscillating piston in the interval of small values of $k$. In the interval of higher values of $k$, the wobbling piston corresponds to the highest value of the normalized acoustic reactance, which implies the greatest fluctuations of the acoustic energy in the near field. However, the knowledge of the radiation impedance of a wobbling piston is important due to the asymmetric distribution of the acoustic field (cf. Eq. (13)).

\section{Concluding remarks}

The Green function for the region above a flat screen, around semi infinite circular cylindrical baffle was used [17] which provided the rigorous solution to the wave equation and enabled calculating the acoustic pressure amplitude of an oscillating and wobbling annular piston embedded concentrically in a perpendicular flat screen surrounding the cylinder. At the assumed velocity distribution of the piston, both the sound pressure and the acoustic impedance are the superpositions of these quantities of the piston oscillating only and the piston wobbling only. For this reason, the acoustic impedance does not depend on the initial phase difference of oscillations and wobbling. It is important to emphasize that the superposition of acoustic impedance is valid in the case of the vibrating piston, whereas in the case of plates and membranes the determination of sound power requires also the use of intermodal impedance coefficients. The acoustic impedance of a wobbling piston does not depend on the spatial location of the nodal diameter, on which the sound pressure distribution obviously depends. The effect of acoustic waves scattering on the cylinder is important since it increases the sound radiation efficiency in the range of low wavenumbers both for the oscillations impedance (agrees with the conclusions presented in [8]) and for the wobbling impedance. Furthermore, the increase in the radius of the cylinder decreases the amplitude of local oscillation of the acoustic resistance and 
reactance as functions of the wavenumber. The effect of the ratio of the outer and inner radii $s$ of the piston on the acoustic impedance of wobbling is the same as in the case of the effect on the acoustic impedance of oscillating (cf. [8]). For practical reasons, however, $s$ should be neither too small $(s \rightarrow 1)$ nor too big $(1 \ll s)$. Based on the numerical analysis it was found that the optimum value of $s$ is about 2.0. It was also found that the sound radiation efficiency of the piston wobbling only is much smaller for small values of the wavenumber than in the case of the piston oscillating only. However, the knowledge of acoustic impedance of the wobbling piston is important due to the asymmetric distribution of the resultant sound pressure.

\section{References}

[1] B. Wyrzykowska, in: Proc. 2nds Conf. on Ultrasonics, Warsaw 1956, p. 99.

[2] R.L. Pritchard, J. Acoust. Soc. Amer. 32, 730 (1960).

[3] D.T. Porter, J. Acoust. Soc. Amer. 36, 1154 (1964).

[4] W. Thompson Jr., J. Sound Vibr. 17, 221 (1971).

[5] P.R. Stepanishen, J. Acoust. Soc. Amer. 56, 305 (1974).

[6] T. Mellow, L. Kärkkäinen, J. Acoust. Soc. Amer. 118, 1311 (2005).

[7] T. Mellow, J. Acoust. Soc. Amer. 120, 90 (2006).

[8] W.P. Rdzanek, W.J. Rdzanek, D. Pieczonka, Archiv. Acoust. 37, 411 (2012).
[9] H. Levine, F.G. Leppington, J. Sound Vibr. 121, 269 (1988).

[10] W.P. Rdzanek, W.J. Rdzanek, K. Szemela, J. Comp. Acoust. 18, 335 (2010).

[11] W.P. Rdzanek, W.J. Rdzanek, K. Szemela, Acta Physica Polonica A 118, 141 (2010).

[12] D. Iwański J. Wiciak, Acta Physica Polonica A 119, 972 (2011).

[13] M.-R. Lee R. Singh, J. Acoust. Soc. Amer. 95, 3311 (1994).

[14] H. Lee R. Singh, J. Sound Vibr. 282, 313 (2005).

[15] J.P. Arenas, J. Comp. Acoust. 16, 321 (2008).

[16] V. Mangulis, J. Acoust. Soc. Amer. 40, 349 (1966).

[17] W.P. Rdzanek, W.J. Rdzanek, A. Różycka, Archives of Acoustics 32, 7 (2007).

[18] A. Sommerfeld, Partial Differential Equations in Physics, Academic Press, New York 1949.

[19] A. Rubinowicz, Reports on Mathematical Physics 2, 93 (1971).

[20] G.N. Watson, A Treatise on The Theory of Bessel Functions, Cambridge University Press, Cambridge 1944.

[21] N.W. McLachlan, Bessel Functions for Engineers, Clarendon Press, Oxford 1955.

[22] A.P. Prudnikov, Yu.A. Brychkov, O.I. Marichev, Integrals Series, Vol. 2: Special Functions, Gordon Breach, New York 1990.

[23] J.W.S. Rayleigh, The Theory of Sound, MacMillan, London 1929.

[24] R.M. Aarts A.J.E.M. Janssen, J. Acoust. Soc. Amer. 113, 2635 (2003). 